\title{
Phronesis
}

\section{Intervention et pratique éducative}

Reflet et/ou révélateur des tensions entre instruction, socialisation et qualification?

\section{Philippe Maubant}

Volume 2, numéro 2-3, avril-juillet 2013

Intervention et pratique éducative : Reflet et/ou tensions entre instruction, socialisation et qualifications?

URI : https://id.erudit.org/iderudit/1018068ar

DOI : https://doi.org/10.7202/1018068ar

Aller au sommaire du numéro

Éditeur(s)

Institut de recherche sur les pratiques éducatives

ISSN

1925-4873 (numérique)

Découvrir la revue

Citer ce document

Maubant, P. (2013). Intervention et pratique éducative : reflet et/ou révélateur des tensions entre instruction, socialisation et qualification? Phronesis, 2(2-3), 1-3. https://doi.org/10.7202/1018068ar d'utilisation que vous pouvez consulter en ligne.

https://apropos.erudit.org/fr/usagers/politique-dutilisation/ 


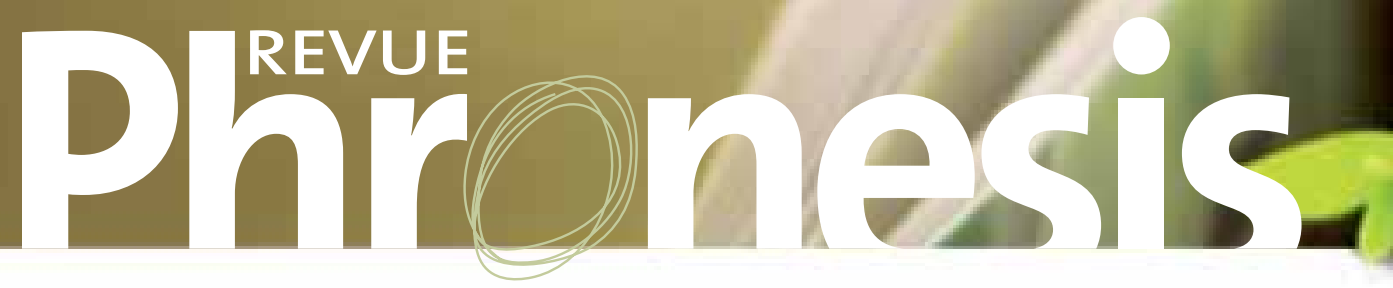

Institut de recherche sur les pratiques éducatives de l'Université de Sherbrooke

Volyme 2, numéro 2-3 | avril-juillet 2013

\section{Intervention et pratique éducative :}

\section{Philippe MAUBANT}

Université de Sherbrooke

2500, boul. de l'Université

Sherbrooke (Québec)

Canada J1K 2R1

Philippe.maubant@usherbrooke.ca

Reflet et/ou révélateur des tensions entre instruction, socialisation et qualification? 
Institut de recherche sur les pratiques éducatives de l'Université de Sherbrooke

Ce numéro a pour objectif de travailler sur ce qui fonde lécole aujourd'hui et ce qui contribue a priori à guider et à accompagner l'action des différents intervenants éducatifs. À la lecture du récent programme de la formation à l’enseignement et du curriculum de l'enseignement au Québec, nous pouvons constater combien la finalité de cette réforme curriculaire est avant tout, selon Lenoir ( $a$ ) en partie d'ordre économique. Il s’agit, à l'instar des autres réformes mises en œuvre dans les pays de l'Organisation de coopération et de développement économique (OCDE) d’adapter les systèmes éducatifs aux exigences imposées par la globalisation. Outre cette mission centrale, le gouvernement du Québec présente également d'autres objectifs, en particulier celui de lutter contre le décrochage scolaire, ce dernier constituant une tierce modalité d'accès à l'objectif économique d'adaptation de la main d'œuvre aux réalités du marché du travail.

Ce numéro de Phronesis vise la mise en évidence de zones de tension entre les trois missions de lécole : instruction, socialisation et qualification. Mais au-delà de ces zones de tension, c'est bien toute la question des conceptions, représentations et perceptions de ces trois missions qui semblent nécessaires d’analyser et de mieux cerner, avant même d’étudier les caractéristiques de cette tension et d’en évaluer leurs effets sur les postures de l'intervention éducative et sur la conception des pratiques des différents acteurs impliqués. Il est donc important d'interroger la manière dont les divers acteurs, parties prenantes des systèmes éducatifs, lisent et interprètent ces trois missions. Si l'on analyse notamment les prescriptions du ministère de l'Éducation, des Loisirs et du Sport du Québec (MELS) par exemple, force est de constater que l'instruction semble constituer la mission centrale de l'école au Québec. Il sagit de faire acquérir par les élèves les savoirs de base : lire, écrire et compter. La mission de socialisation, quant à elle, est définie autour de l’expression de "vivre ensemble ». La mission de qualification n’est pas davantage plus précise puisquelle semble renvoyer comme un allant-de soi à l'impérieuse nécessité d’adapter la formation à lemploi, tout en rappelant l’ambition d’une société du savoir, garante du développement économique et social.

Les enseignants mesurent de toute évidence la puissance de l'objectif d'instruction, celui de transmettre des savoirs. Mais l'une des questions centrales qu'il convient de poser, c'est celle d'identifier de quels savoirs il s'agit. Quels sont les savoirs à transmettre? Pourquoi enseigner ces savoirs et pas d’autres? De quels savoirs parle-t-on? Comment les enseignants les déterminent-ils et les sélectionnent-ils dans et par un curriculum dont ils percevront rapidement la complexité voire l’ambiguïté épistémologique? À quelles fins est perçue l'intervention éducative et quelles pratiques découlent de celles-ci? En interrogeant la mission d'instruction, c'est donc bien toute la question des savoirs, et plus généralement du rapport au Savoir et aux savoirs qui se pose, en particulier si lon considère que les différentes lectures de cette mission d'instruction mâtinent ces rapports au Savoir et aux savoirs des différents acteurs impliqués dans la situation d'enseignement-apprentissage.

Quant à la socialisation, elle renvoie aujourd'hui à différentes théories, à diverses perspectives épistémologiques. Elle s’enracine aussi dans différentes tentatives de typologisation. Comme le note Lenoir ( $a p$ ), cette variété de figures et de configurations de la socialisation dépend des liens entre les différentes structures sociales qu'il s'agisse des familles, des institutions, des groupes économiques, ou encore des services publics comme lécole par exemple. Que dire des organismes communautaires et gouvernementaux qui agissent en partenariat avec les institutions scolaires? En affirmant cela, nous rappelons combien la socialisation échappe à lécole et plus généralement aux différents temps éducatifs pour être revisitée au travers des multiples situations rencontrées par l'individu. Qu'attend-on aujourd'hui de l'École, outre la transmission de savoirs? Qu’elle accompagne le jeune dans un parcours visant la réussite. Mais de quelle réussite s’agit-il ? Une réussite scolaire avant toute chose. Mais aussi une réussite éducative, professionnelle et sociale. De quelles manières l'École peut-elle atteindre cet objectif, elle qui se trouve au cœur des tensions sociales et au centre des luttes individuelles et collectives pour la reconnaissance? Socialiser, certes, mais pourquoi et pour qui ? Comment soutenir cet objectif de socialisation au travers des différentes situations d'enseignement-apprentissage? Peut-on apprendre à socialiser? Peut-on apprendre et enseigner des savoirs ou des compétences de socialisation? Les compétences sociales sont-elles constitutives de la socialisation ou sont-elles présentes dans et par d'autres savoirs scolaires? Comment intégrer les familles et la communauté dans la réalisation conjointe et partagée de cette ambition de socialisation, sans renier les interprétations, sans exclure les pra- 
tiques collectives socialisatrices des familles, des groupes et des communautés?

La qualification, quant à elle, se doit dêtre interrogée sur ses visées et sur les manières selon lesquelles chaque acteur du système éducatif tente d'y répondre. Encore convient-il, là encore, de s'entendre sur ce terme en analysant les retombées attendues d'une telle mission : adaptation à l'emploi, adaptation des savoirs et des compétences aux réalités des postes de travail, insertion professionnelle, lutte contre le chômage ou le décrochage scolaire, soutien au développement professionnel, autant d’attentes incarnées semble-t-il par lobjectif de qualification. À nouveau, au-delà d'un allant de soi qui tend à poser comme postulat la visée « qualifiante » de l'École, de quelle qualification parle-t-on? Comment concilier la visée séculaire d'une éducation émancipatrice s’appuyant sur l’universalité de la culture et celle, plus récente, confinant léducation à des enjeux d’adaptation de la main d’ouvre au marché du travail, et donc réduisant celle-ci à une finalisation opératoire des savoirs scolaires? Comment intégrer cette visée de qualification dans les situations d'enseignement-apprentissage? Quelles perspectives épistémiques fonderont l'intervention éducative et sur quoi s’encreront les pratiques? Comment former les futurs enseignants à cette mission? Enfin, comment identifier, au sein du curriculum, des savoirs ou des compétences soutenant l'ambition qualifiante de l'École? Conviendraitil de transposer tous les savoirs scolaires de telles manières à ce qu'ils prennent sens au regard d'un usage social et professionnel? Dès lors, ne risquons-nous pas de renforcer une réalité croissante de deux écoles, l'une préparant à la mise en emploi, la seconde permettant l'accès à l'universalité de la culture?

Si l'on vise à mettre à jour quelques grandes questions et enjeux constitutifs de ces trois missions et surtout si l'on cherche à mettre en évidence les points d'intersection voire les zones de tension entre ces trois missions, il semble possible d’ores et déjà de repérer quatre grands types d'enjeux :

1. L'enjeu des finalités de tout système scolaire. Autrement dit, que cherche-t-on dans l'affirmation de chacune de ces trois missions? L'une est-elle un préalable aux deux autres?

2. Second enjeu, celui de l'interprétation par les différents acteurs concernés de chacun de ces objectifs. Quelles significations donnent les enseignants, les différents intervenants éducatifs, les familles ou d'autres acteurs aux objectifs d'instruction, de socialisation ou de qualification?

3. Troisième enjeu, celui des modalités de mise en œuvre de ces objectifs : de quelles manières et sous quelles conditions les intervenants éducatifs et socio-éducatifs conçoivent et mettent en œuvre ces trois missions? Quels sont les démarches de mise en œuvre et les instruments dévaluation qu'ils se donnent pour vérifier l'atteinte de ces objectifs?

4. Dernier enjeu, celui des résistances, voire des freins identifiés au travers de la mise en place des ces trois missions, et celui des stratégies déployées par l'institution, les acteurs éducatifs et socio-éducatifs et les usagers pour les contourner, voire les dépasser?

En interrogeant l'intervention et les pratiques éducatives comme révélatrices des tensions entre instruction, socialisation et qualification, les contributeurs à ce numéro ont été invités à répondre aux principales questions suivantes :

1. De quelles manières la figure didactique et pédagogique de l'intervention éducative se trouve-t-elle influencée par ces tensions entre instruire, socialiser et qualifier?

2. Comment les disciplines, les savoirs disciplinaires et non disciplinaires sont-ils réinterrogés au regard de leurs finalités à rendre intelligible le monde et quelles sont leurs formes de légitimité?

3. Comment les curriculums de formation à l'enseignement et les dispositifs de formation «professionnalisants » prennent-ils en compte ces trois missions?

4. De quelles manières ces trois missions modifient-elles les rapports au Savoir et aux savoirs des différents acteurs?

5. Comment les familles et les acteurs de la communauté perçoivent, s’adaptent ou résistent-elles aux tensions entre les trois missions de lécole?

6. Comment ces tensions entre instruction, socialisation et qualification modifient-elles la problématique du rapport à lécole, du rapport à la communauté et au monde 